www.jmscr.igmpublication.org

Index Copernicus Value: 79.54

ISSN (e)-2347-176x ISSN (p) 2455-0450

crossrefDOI: https://dx.doi.org/10.18535/jmscr/v7i1.135

\title{
A Multi-Centered Study to Evaluate the Efficacy and Safety of Amitriptyline and Mecobalamine in the Management of Low Back Pain and Lumbar Radiculopathy
}

\author{
Authors \\ Dr Mayuresh Dilip Kiran ${ }^{1}$, Shaheen Naseem Sheikh ${ }^{2}$, Lalit Jeeevan Pawaskar ${ }^{3}$ \\ ${ }^{1}$ Vice-President, Medical Services and Pharmacovigilance, Centaur Pharmaceuticals Pvt. Ltd \\ ${ }^{2}$ Research Associate, Pharmacovigilance, Centaur Pharmaceuticals Pvt. Ltd \\ ${ }^{3}$ Executive, Pharmacovigilance, Centaur Pharmaceuticals Pvt. Ltd \\ *Corresponding Author \\ Dr Mayuresh Dilip Kiran \\ Vice-President, Medical Services and Pharmacovigilance., Centaur Pharmaceuticals Pvt. Ltd. \\ Mumbai, India \\ Mobile No.+91-9820068567, Email: mayuresh_kiran@rediffmail.com
}

\begin{abstract}
Introduction: Low Back Pain (LBP) is a pain and disability comfort localized below costal margins and above the inferior gluteal folds with or without referred pain in legs. Lumbar Radiculopathy (LR) is a term used to describe pain caused by compression or irritation of nerve root in the low back. An FDC of Amitriptyline a neuroanalgesia and Mecobalamine (Vitamin $B_{12}$ ) a damage nerve rejuvenator have an important role in management of LBP and LR caused by Neuropathic pain.

Methodology: Of 400 registered, 339 patients completed the study. Efficacy assessment was made by reduction in mean VAS score and percentage of patients with $\geq 50 \%$ reduction in LBP and LR was calculated at conclusion visit and used to determine the corresponding NNT. Safety assessment was made by investigating the adverse events during the study.

Results: Reduction in mean VAS score, in LBP patients was from 8.01 (baseline) to 4.18 (day 30) and 1.74 (day 45) and in LR patients reduction was from 7.24 (baseline) to 4.80 (day 30) and 3.20 (day 45). Nearly all the patients had experience $\geq 50 \%$ reduction in LBP and LR. NNT score was calculated by using risk reduction parameter at conclusion visit. Overall 116 patients experience adverse events and were of mild to moderate intensity.

Conclusion: The combination of Amitriptyline and Mecobalamine was safe, efficacious and well-tolerated in management of Low back pain (LBP) and Lumbar Radiculopathy (LR).

Keywords: Low back pain, Lumbar radiculopathy, Neuropathic pain, Amitriptyline, Mecobalamine, VAS score and NNT score.
\end{abstract}

\section{Introduction}

Neuropathic Pain (NP) is a medical condition develops after a lesion or disease affecting the somatosensory nervous system either at peripheral or central level. ${ }^{[1],[2]}$ Diabetic neuropathy, trigeminal neuralgia, post-spinal cord injury pain, and post-herpetic neuralgia are characteristic examples of NP. NP serves a significant role in 
the pathogenesis of numerous diseases related to the spine. However, and is based on a characteristic symptom profile and diagnostic tests, the diagnosis of NP remains clinical. ${ }^{[3]}$

Low Back Pain (LBP) is defined as pain and disability comfort localized below costal margins and above the inferior gluteal folds with or without referred pain in legs. ${ }^{[4],[5]}$ LBP is the most common musculoskeletal pain and one of the most widespread public health related problem worldwide. The differential for LBP is broad and amongst other diagnoses, should include Lumbar LR (LR). LR is a term used to describe pain caused by compression or irritation of nerve root in the low back. It can be caused by degeneration of the spinal vertebra, lumbar disc herniation and narrowing of the foramen from which the nerves exit the spinal canal. However, any process that stimulates the irritation of the spinal nerve can cause radicular symptoms. ${ }^{[6]}$

Multiple studies have yielded variable results on the prevalence of LBP and thus the epidemiology of LR is unclear. This is likely because the conditions that cause lower extremity Back Pain are often poorly defined. However, the incidence of LBP is estimated between 13 to $31 \%$. Moreover, the incidence of radicular symptoms in patients with LBP ranges from 12 to $40 \%$. Patients with chronic LBP account for $80 \%$ to $90 \%$ of all health care expenditures. ${ }^{[6]}$

Despite the introduction of new treatments, the management of patients with NP remains a challenge. In clinical practice, patients with NP include those with spinal pain, often receive suboptimal treatment. ${ }^{[7]}$ One of the treatment option available for LBP and LR caused by NP is Drug Therapy. Anti-depressants are commonly prescribed drug and their use is increasing for the treatment for LBP and LR. ${ }^{5}$ The two main groups used as Drug Therapy is Tricyclic anti-depressants (TCAs) and Selective Serotonin Re-uptake Inhibitors (SSRIs). ${ }^{[5],[8]}$ However, there is evidence to indicate that the TCAs and SSRIs have an effect on endogenous opioid and peripheral system. ${ }^{[5],[9]}$ Anti-depressants at significantly lower doses are used to treat NP than those which are used for depression. ${ }^{[5],[10]}$ A high quality trial by Atkinson, which performed a head to head comparison of TCAs and SSRIs, reported a greater reduction in pain intensity with low doses of TCAs than with all doses SSRIs. Therefore Low dose anti-depressants are a common treatment for LBP and LR. ${ }^{[5], ~[11] ~}$

Amitriptyline, a Tricyclic antidepressants used as first- line treatment, also has analgesic properties and are effective in the treatment of neuropathic pain for many years. The mechanism of action lies on inhibiting sodium channels that allows the stabilization of neuronal peripheral level and modulation of neuronal hyperactivity at central level, also blocks the reuptake of norepinephrine and serotonin to pre-synaptic level, limiting the hyperalgesia induced by N-methyl-D-aspartate (NMDA) agonists, anti-histamine action on the $\mathrm{H} 1$ and $\mathrm{H} 2$ receptors, blockade of alpha receptors that can eliminate pain. ${ }^{[12],[13],[14]}$

Cobalamine has 4 analogues; cyanocobalamine, hydroxycobalamin, 5-deoxyadenosylcobalamin and mecobalamin, out of which mecobalamin accounts for $90 \%$ of total cobalamine, suggesting its close relationship with nervous system. Thus, it will be more be more appropriate to consider it in neuropathy rather than other analogues. ${ }^{[15]}$ Mecobalamin is the neurologically active, most bioavailable and best utilization form of Vitamin $\mathrm{B}_{12 .}{ }^{[16]}$ Mecobalamine helps in nerve rejuvenation by promoting myelination, restoring diminished neurotransmitter levels and axonal regeneration. It also offers protection against glutamate-induced neurotoxicity. ${ }^{[17]}$

As neuroanalgesia, Amitriptyline is of a particular value in the management of NP caused by LBP and LR. Mecobalamine is the physiologically most active from of Vitamin $B_{12}$. Mecobalamine restores diminished neurotransmitter levels, causes nerve rejuvenation by promoting myelination, and axonal regeneration. The combination of Amitriptyline and Mecobalamine can be valuable assets in the management of NP. However, due to paucity of available data on the 
efficacy and safety of the combination of Amitriptyline and Mecobalamine this is the Indian clinical study (AMNEST) which evaluate the efficacy and safety of Amitriptyline and Mecobalamine combination in management of NP.

\section{Methodology}

Phase IV, multi-centered, clinical study was conducted in 250 centres all over India. Total 400 patients were recruited, out of which 339 patients completed the study and 61 patients lost to followup. From 339 patients, 213 and 126 patients were of LBP and LR respectively.

\section{Inclusion and Exclusion Criteria}

The study includes patients of both the genders, age 18-65 years. Patients with confirmed diagnosis of Neuropathic pain caused by LBP and LR were included in the study. If the patient is diabetic, treatment with anti-diabetics drugs has been given. Patients with LBP and LRcaused by NP is not controlled by any other drug or treatment. Only patients who are participated and strictly adhere to protocol were mustered for this phase IV clinical study. Patients having hypersensitivity to any ingredient of the formulation were excluded from the study. Patients taking MAO-inhibitors were excluded or history of urinary retention due to benign prostatic hyperplasia were excluded from the study. Patients with renal/hepatic impairment or with any evidence of psychological disorder were also excluded from the study.

\section{Study Intervention}

Patients were instructed to take Study drug - A film coated bilayered tablet contains Amitriptyline $10 \mathrm{mg}$ (Immediate release) and Mecobalamine $1500 \mathrm{mcg}$ (Sustained release). The study drug was provided free of cost by the sponsor to recruited patients. Patients were instructed to the study drug (1 tablet) at night for study duration of 45 days.

\section{Study Procedure}

The study duration to determine the safety and efficacy of study drug combination (Amitriptyline and Mecobalamine) in management of neuropathic pain caused by LBP and LR was kept 45 days. Neuropathic pain patients satisfying the inclusion/exclusion criteria were recruited for the study. Physical examination includes blood pressure plus, rate and respiratory rate was conducted and case history was taken by the investigators. Five blister packs (10 tablets each blister) was dispensed to patients and were advised to take a dose of one tablet at night. Determination of efficacy in patients were evaluated by using Visual Analogue Scale (VAS) score. The VAS score of patients was noted on Baseline visit (V1) on day 1, Re-evaluation visit (V2) on day 30 and Conclusion visit (V3) on day 45. As compared to the baseline, the change in VAS score at V2 and V3 was statistically evaluated. Any adverse event or adverse drug reaction occurred during the study stretch of 45 days was noted by patients as instructed by the investigators. Patients were requested to withdraw the study medication in case of any severe adverse event or drug reaction by the investigator. Number Needed to Treat (NNT) is a measure to rank the efficacy of the treatment including analgesics. NNT is use to relate the efficacy of treatment in chronic pain and is defined as the number of patients that need to treated for a given period of time to achieve beneficial outcome i.e. $50 \%$ pain relief. NNT close to 1 means treatment is $100 \%$ effective. Basically smaller the NNT, fewer the number of patients needed to treat to achieve a beneficial effect.

\section{Concomitant Therapy}

During the study duration patients were instructed not to use any other analgesics apart from the study medication. Any non-pharmacological therapy to attenuate pain was allowed during the study duration. Any use of concomitant medications was also noted.

\section{Efficacy Assessment}

The primitive assessment was done by determining the reduction in the VAS score on an eleven-point scale ( 0 to 10$)$ where 0 means no pain and 10 means worst possible pain. The subsidiary assessment was done to evaluate 
percentage of patients with $\geq 50 \%$ reduction in pain caused by LBP and LR from baseline were calculated at V3 i.e. day 45 and this value was used to access the corresponding NNT. Hence, the NNT was calculated using risk reduction.

\section{Safety Assessment}

Any adverse event or adverse drug reaction if occurred, patients were examined at each visit and was noted in the case report form (CRF) by the investigator. The adverse events are classified into serious and non- serious adverse events Naranjo's scale of probability was used to classify the adverse event as drug related or non-drug related. Investigator can be withdrawn from study if adverse event found to be serious and necessary follow-up and treatment was provided till the adverse events resolves.

\section{Regulatory Matters}

The study combination has been approved for manufacturing and marketing in 2005. The combination is available under various brands but is classified as schedule $\mathrm{H}$ drug in India, i.e. to be sold in presence of prescription of registered medical practitioners only. All the patients who participated in the study has read and signed the

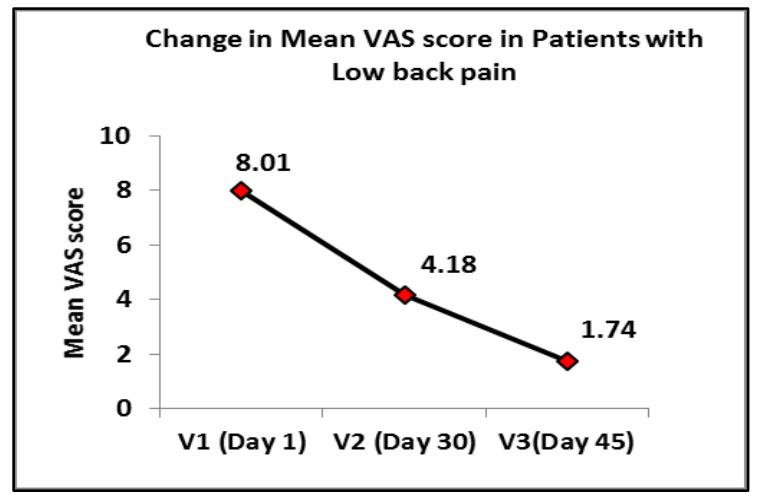

Figure 1: Change in Mean VAS score in LBP patients

Similarly in patients of LR the mean VAS score at baseline visit (V1) was found to be 7.42 which reduced to $4.80(35.3 \%)$ at V2 and further reduced to $3.20(56.81 \%)$ at V3 (Figure 3). Percentage of informed consent form voluntarily. The protocol, case record form (CRF), informed consent form (ICF), undertaking by investigators, $\mathrm{CV}$ and medical registration certificates (including postgraduation certificates) of investigators and ethics committee registration certificates were submitted to the office of Central Drugs Standard Control Organization (CDSCO).

\section{Results}

Total 339 patients were analysed based on inclusion and exclusion criteria, out of which 213 and 126 patients were diagnosed with LBP and LR respectively. The two main outcome of the study is change in mean VAS score from baseline visit (V1) at day 1 to conclusion visit (V3) at day 45 and percentage of patients observed with $\geq 50 \%$ reduction in pain responds to study medication.

In patients of LBP, the mean VAS score at baseline visit (V1) was found to be 8.01 which reduced to $4.18(48 \%)$ at $\mathrm{V} 2$ and further reduced to $1.74(78 \%)$ at V3 (Figure 1). Percentage of patients with $\geq 50 \%$ reduction in pain during visit V2 and V3 was found to be $76 \%$ and $92.30 \%$ respectively (Figure 2).

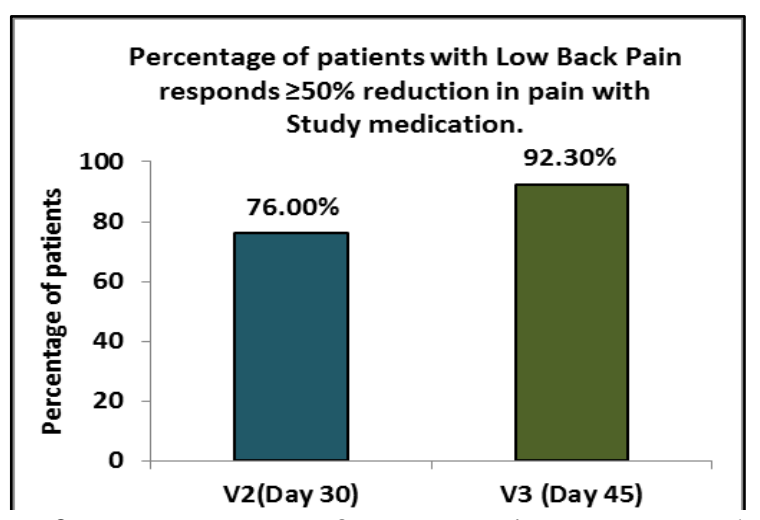

Figure 2: Percentage of LBP patients responds $(\geq 50 \%$ reduction in pain) to Study medication

patients with $\geq 50 \%$ reduction in pain during visit V2 and V3 was found to be $47.05 \%$ and $76.47 \%$ respectively (Figure 4). 


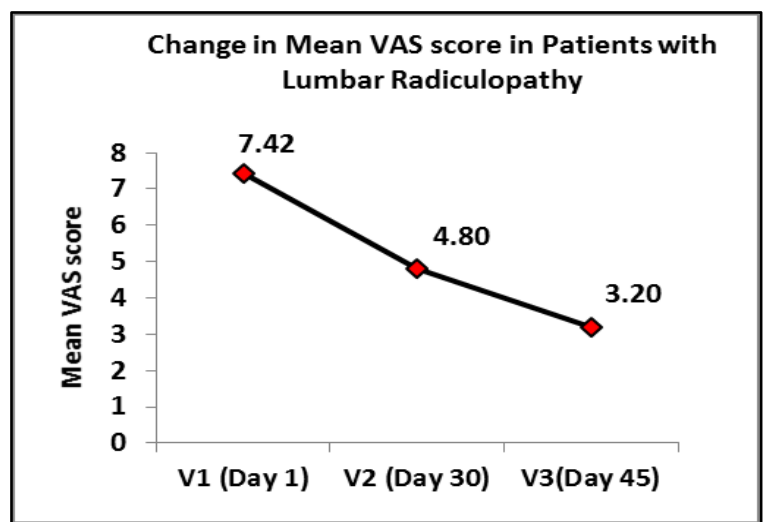

Figure 3: Change in Mean VAS score in LR patients

NNT of study medication in patients with LBP and LR was calculated using percentage of patients observed with $\geq 50 \%$ reduction in pain at

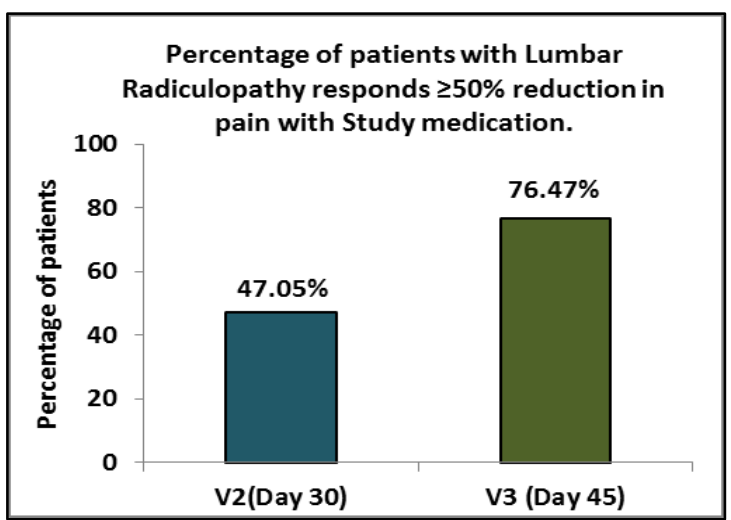

Figure 4: Percentage of LR patients responds ( $\geq 50 \%$ reduction in pain) to Study medication

V3 (Table 1). NNT close to 1 means that a treatment is nearly $100 \%$ effective.

Table no. 1: NNT of Study medication in Patients of LBP and LR.

\begin{tabular}{|l|c|c|c|c|}
\hline Sr.no & $\begin{array}{c}\text { Type of } \\
\text { Pain }\end{array}$ & $\begin{array}{c}\text { Percentage of patients with } \geq \mathbf{5 0 \%} \\
\text { reduction in pain at Conclusion visit (V3) }\end{array}$ & $\begin{array}{c}\text { Risk Reduction } \\
\text { (RR) }\end{array}$ & $\begin{array}{c}\text { Number needed to } \\
\text { treat (NNT) }\end{array}$ \\
\hline 1. & LBP & 92.30 & 0.923 & 1.08 \\
\hline 2. & LR & 74.47 & 0.764 & 1.30 \\
\hline
\end{tabular}

No serious adverse events was observed during the study duration. The overall incidence of reported study drug related adverse effects were seen in $34.21 \%$ patients. The list of adverse events with the number of episodes is mentioned in Table 2.

Table no. 2: Adverse events, no. of episodes, no. of patients and percentage of patients experience from total population

\begin{tabular}{|l|c|c|}
\hline Adverse event & No. of patient & Percentage of patients \\
\hline Sedation and Drowsiness & 81 & 23.89 \\
\hline Dizziness & 15 & 4.42 \\
\hline Dry mouth & 32 & 9.43 \\
\hline Constipation & 17 & 5.01 \\
\hline GI irritation & 28 & 8.25 \\
\hline Total & $\mathbf{1 1 6}$ & $\mathbf{3 4 . 2 1}$ \\
\hline
\end{tabular}

\section{Discussion}

Neuroanalgesics with neuron rejuvenator's combination are available in the market and are quite popular. "AMNEST" is the first Indian study determining the safety and efficacy of Amitriptyline and Mecobalamine combination in management of LBP and LR caused by NP in Indian patients. The strength of the study is both the parameters which leads to reduction in NP i.e. change in mean VAS score and NNT were clinically studied over the study duration of 45 days.
At baseline visit (V1) o day 1, the mean VAS score for LBP and LR was found to be 8.01 and 7.42 which reduced to 4.18 and 4.80 at reevaluation visit (V2) on day 30 and further reduced to 1.74 and 3.20 at conclusion visit (V3) on day 45 respectively. LBP and LR patients with $\geq 50 \%$ reduction in pain was found to be $76 \%$ and $47.05 \%$ at re-evaluation visit (V2) on day 30 and $92.30 \%$ and $76.47 \%$ at conclusion visit (V3) on day 45 respectively. Patients were also demonstrated on NNT for study medication and was calculated by using percentage of patients 
with $\geq 50 \%$ reduction in pain at $\mathrm{V} 3$. The Risk reduction for LBP and LR was found out to be 0.923 and 0.747 respectively. NNT is inverse of risk reduction value and close to 1 means treatment is $100 \%$ effective. Hence with study medication NNT was found out to be 1.08 and 1.30 for LBP and LR respectively. In 116 patients, study drug related adverse events were reported. Sedation and Drowsiness (23.89\%), Dizziness (4.42\%), Dry mouth $(9.43 \%)$, Constipation $(5.01 \%)$ and GI irritation $(8.25 \%)$ was the documented adverse event in this study and were of mild intensity affecting $34.21 \%$ of the study population.

Kalita, J., et al. ${ }^{[18]}$ has conducted an open-labeled, singled center, investigators initiated randomised controlled trial to report the safety and efficacy of Pregabalin (PG) and Amitriptyline (AT) in chronic low backache (CLBA). Patients with CLBA, age 15-65 years without specific cause and significant neurological deficit were included. Severity of pain was measured by Visual Analogue Scale (VAS) and disability by Oswestry Disability Index (ODI). Patients were followed up at 6 and 14 weeks respectively and their VAS score, ODI and side effect were noted. The main outcome of the study was pain relief i.e. patients response $>50 \%$ improvement in VAS score at 14 weeks and ODI reduction (>20\%) and side effects. Total 200 patients with CLBA were randomized in to two groups PG $(n=97)$ and AT $(n=103)$ by using random numbers. The VAS score and ODI improved significantly following PG and AT at 6 and 14 weeks compared to baseline. The improvement in pain $(\mathrm{PG}=39.2 \%$ Vs $\mathrm{AT}=57.3 \%$; $\mathrm{P}=0.01)$ and disability $(\mathrm{PG}=49.5 \%$ Vs $\mathrm{AT}=65 \%$; $\mathrm{P}=0.03$ ) however was more in AT group. The composite side effects were noted and found to be similar in both groups. AT and PG are effective, but AT reduced pain and disability significantly compared to PG in CLBA.

Dongre Yasmin U., et al ${ }^{[19]}$ as conducted openlabeled, multicentre, single-arm, prospective, observational clinical study for the duration of 14 days in Indian patients. Patients received fixed dose combination of sustained-release pregabalin combined with immediate release methylcobalamin. Data was collected for reduction in pain and other positive and negative symptoms associated with NP, including numbness or tingling, hyperesthesia, paresthesia, muscle weakness, burning sensation, impairment of movement, and sleep disturbances. Pain intensity was measured on a $0-10$ point Visual Analogue Scale (VAS) $(0=$ no pain and $10=$ worst pain ever). The drug safety was also evaluated throughout the study duration of 14 days. The data was analyzed using suitable statistical methods. The overall $72.3 \%$ reduction was observed in mean VAS score over 14 days. The reduction in mean VAS score was significant as early as the first week. Both positive and negative symptoms of peripheral NP were significantly improved in $>50 \%$ patients within the 2 weeks. Giddiness (4.7\%), sedation (3.6\%), dizziness (2.9\%), drowsiness $(2.3 \%)$, and nausea $(2.3 \%)$ were the most commonly observed adverse effects noted during the study. The overall efficacy and tolerability was rated as good to excellent by $>95 \%$ of the investigators and patients. Therefore FDC of sustained-release pregabalin and methylcobalamin significantly reduced NP, with significant improvement in both the positive and negative symptoms associated with NP, in Indian patients and was well tolerated.

\section{Conclusion}

The drug combination of Amitriptyline and Mecobalamine was safe, efficacious and welltolerated in management of Low back pain (LBP) and Lumbar radiculopathy (LR) due to Neuropathic pain (NP).

\section{Acknowledgements and Disclosures}

"AMNEST" study was conducted as a part of Pharmacovigilance activity for Amnurite Tablet marketed by Centaur Pharmaceuticals Pvt. Ltd. in accordance with Pharmacovigilance Program of India (PvPI). 


\section{Reference}

1. Saxena, A. K., S. Nath, and R. Kapoor. "Diabetic Peripheral Neuropathy: Current Concepts and Future Perspectives." Journal of Endocrinology and Diabetes 2.5 (2015): 1-8.

2. Baron, Ralf, et al. "Peripheral neuropathic pain: a mechanism-related organizing principle based on sensory profiles." Pain 158.2 (2017): 261.

3. Park, Si Young, et al. "Neuropathic pain components in patients with lumbar spinal stenosis." Yonsei medical journal 56.4 (2015): 1044-1050.

4. Elders, Leo AM, and Alex Burdorf. "Prevalence, incidence, and recurrence of low back pain in scaffolders during a 3year follow-up study." Spine 29.6 (2004): E101-E106.

5. Urquhart, Donna M., et al. "Is low-dose amitriptyline effective in the management of chronic low back pain? Study protocol for a randomised controlled trial." Trials 17.1 (2016): 514.

6. Alexander, Christopher, and Scott Dulebohn. "Lumbosacral Radiculopathy." (2017).

7. Saldaña, María Teresa, et al. "Patientreported-outcomes in subjects with painful lumbar or cervical radiculopathy treated with pregabalin: evidence from medical practice in primary care settings." Rheumatology international 30.8 (2010): 1005-1015.

8. Micó, Juan A., et al. "Antidepressants and pain." Trends in pharmacological sciences 27.7 (2006): 348-354.

9. Goldenberg, Don L. "Pharmacological treatment of fibromyalgia and other chronic musculoskeletal pain." Best Practice \& Research Clinical Rheumatology 21.3 (2007): 499-511.
10. McQuay, H. J., et al. "A systematic review of antidepressants in neuropathic pain." Pain 68.2-3 (1996): 217-227.

11. Atkinson, J. Hampton, et al. "Efficacy of noradrenergic and serotonergic antidepressants in chronic back pain: a preliminary concentration-controlled trial." Journal of clinical psychopharmacology 27.2 (2007): 135-142.

12. Davis, Jonathan L., et al. "Peripheral diabetic neuropathy treated with amitriptyline and fluphenazine." Jama 238.21 (1977): 2291-2292.

13. Moore, R. Andrew, et al. "Amitriptyline for neuropathic pain and fibromyalgia in adults." Cochrane Database Syst Rev 12.12 (2012).

14. Bryson, Harriet M., and Michelle I. Wilde. "Amitriptyline." Drugs ～\& aging 8.6 (1996): 459-476.

15. Yaqub, Basim A., Abdulaziz Siddique, and Riad Sulimani. "Effects of methylcobalamin on diabetic neuropathy." Clin Neurol Neurosurg 94.2 (1992): 105-111.

16. Thanon, Imad A. J., Ashraf H. Ahmed, and Bashar S. Mahmood. "Efficacy of mecobalamin (Methylcobalamin) in the treatment of diabetic peripheral neuropathy "Medical Journal of Babylon 2.3 (2005): 330-335.

17. Zhang, Ming, et al. "Methylcobalamin: a potential vitamin of pain killer." Neural plasticity 2013 (2013).

18. Kalita, J., et al. "An open labeled randomized controlled trial of pregabalin versus amitriptyline in chronic low backache." Journal of the neurological sciences 342.1 (2014): 127-132.

19. Dongre, Yasmin U., and Onkar C. Swami. "sustained-release pregabalin with methylcobalamin in neuropathic pain: an Indian real-life experience." International journal of general medicine 6 (2013): 413. 